CUBO A Mathematical Journal

Vol.21, No 02, (41-49). August 2019

\title{
Totally umbilical proper slant submanifolds of para-Kenmotsu manifold
}

\author{
M.S. Siddesha ${ }^{1}$, C.S. BAGEWAdi ${ }^{2}$ AND D. Nirmala ${ }^{3}$ \\ ${ }^{1}$ Department of mathematics, \\ New Horizon College of Engineering, \\ Bangalore, India \\ ${ }^{2,3}$ Department of Mathematics, \\ Kuvempu University, \\ Shankaraghatta, Shimoga, Karnataka, India \\ mssiddesha@gmail.com, prof_bagewadi@yahoo.co.in, nirmaladraj14@gmail.com
}

\begin{abstract}
In this paper, we study slant submanifolds of a para-Kenmotsu manifold. We prove that totally umbilical slant submanifold of a para-Kenmotsu manifold is either invariant or anti-invariant or dimension of submanifold is 1 or the mean curvature vector $\mathrm{H}$ of the submanifold lies in the invariant normal subbundle.
\end{abstract}

\section{RESUMEN}

En este paper estudiamos subvariedades inclinadas en variedades para-Kenmotsu. Demostramos que una subvariedad inclinada en una variedad para-Kenmotsu totalmente umbilical es invariante, o anti-invariante, o una subvariedad de dimensión 1, o el vector de curvatura media $\mathrm{H}$ de la subvariedad vive en el fibrado normal invariante.

Keywords and Phrases: para-Kenmotsu manifold; totally umbilical; slant submanifold. 2010 AMS Mathematics Subject Classification: 53C25, 53C40, 53D15. 


\section{Introduction}

The study of submanifolds of an almost contact manifold is one of the utmost interesting topics in differential geometry. According to the behaviour of the tangent bundle of a submanifold with respect to action of the almost contact structure $\phi$ of the ambient manifold, there are two well known classes of submanifolds, namely, invariant and anti-invariant submanifolds. Chen [4, introduced the notion of slant submanifolds of the almost Hermitian manifolds. The contact version of slant submanifolds were given by Lotta [12. Since then many research articles have been appeared on the existence of different contact and lorentzian manifolds (See. 1, 3, 7, 14, 15]).

Motivated by the above studies, in the present paper we study slant submanifolds of almost para-Kenmotsu manifold and give a classification of results. Also we prove that totally umbilical slant submanifolds of para-Kenmotsu manifolds are totally geodesic.

The paper is organized as follows: In section 2, we review some basic concepts of para-Kenmotsu manifold and submanifold theory. Section 3 is the main section of this paper. In this section we give the classification result of totally umbilical slant submanifolds of para-Kenmotsu manifold. Further, we prove that totally umbilical slant submanifolds of a para-Kenmotsu manifold are totally geodesic.

\section{Preliminaries}

Let $\tilde{M}$ be a $(2 m+1)$-dimensional smooth manifold, $\phi$ a tensor field of type $(1,1), \xi$ a vector field and $\eta$ a 1 -form. We say that $(\phi, \xi, \eta)$ is an almost para contact structure on $\tilde{M}$ if [18]

$$
\begin{aligned}
& \phi \xi=0, \quad \eta \cdot \phi=0, \quad \operatorname{rank}(\phi)=2 \mathrm{~m}, \\
& \phi^{2}=\mathrm{I}-\eta \otimes \xi, \quad \eta(\xi)=1 .
\end{aligned}
$$

If an almost paracontact manifold admits a pseudo Riemannian metric $g$ of signature $(m+1, m)$ satisfying

$$
g(\phi \cdot, \phi \cdot)=-g+\eta \otimes \eta
$$

called almost para contact metric manifold. Examples of almost para contact metric structure are given in [6] and 9 .

Analogous to the definition of Kenmotsu manifold [10, Welyczko [17] introduced para-Kenmotsu structure for three dimensional normal almost para contact metric structures. The similar notion called p-Kenmotsu structure appears in the Sinha and Sai Prasad [16].

Definition 2.1. An almost para contact metric manifold $\mathrm{M}(\phi, \xi, \eta, \mathrm{g})$ is para-Kenmotsu manifold if the Levi-Civita connection $\tilde{\nabla}$ of $\mathrm{g}$ satisfies

$$
\left(\tilde{\nabla}_{X} \phi\right) Y=g(\phi X, Y) \xi-\eta(Y) \phi X,
$$


for any $\mathrm{X}, \mathrm{Y} \in \mathrm{\chi}(\mathrm{M})$, (where $\chi(\mathrm{M})$ is the set of all differential vector fields on $\mathrm{M}$ ).

From (2.4), we have

$$
\tilde{\nabla}_{X} \xi=X-\eta(X) \xi .
$$

Assume $M$ is a submanifold of a para-Kenmotsu manifold $\tilde{M}$. Let $g$ and $\nabla$ be the induced Riemannian metric and connections of $M$, respectively. Then the Gauss and Weingarten formulae are given respectively, by

$$
\begin{aligned}
\tilde{\nabla}_{X} Y & =\nabla_{X} Y+\sigma(X, Y), \\
\tilde{\nabla}_{X} N & =-A_{N} X+\nabla_{X}^{\perp} N,
\end{aligned}
$$

for all $X, Y$ on $T M$ and $N \in T^{\perp} M$, where $\nabla^{\perp}$ is the normal connection and $A$ is the shape operator of $M$ with respect to the unit normal vector $N$. The second fundamental form $\sigma$ and the shape operator $A$ are related by:

$$
g(\sigma(X, Y), N)=g\left(A_{N} X, Y\right) .
$$

Now for any $\mathrm{X} \in \Gamma(\mathrm{TM})$ and $\mathrm{V} \in \Gamma\left(\mathrm{T}^{\perp} \mathrm{M}\right)$, we write

$$
\begin{aligned}
& \phi X=P X+F X, \\
& \phi V=p V+f V .
\end{aligned}
$$

For $X, Y \in \Gamma(T M)$, it is easy to observe from (2.1) and (2.9) that

$$
g(P X, Y)=-g(X, P Y)
$$

The covariant derivatives of the endomorphisms $\phi, P$ and $F$ are defined respectively as

$$
\begin{aligned}
\left(\tilde{\nabla}_{X} \phi\right) Y & =\tilde{\nabla}_{X} \phi Y-\phi \tilde{\nabla}_{X} Y, \quad \forall X, Y \in \Gamma(T \tilde{M}), \\
\left(\tilde{\nabla}_{X} \mathrm{P}\right) Y & =\nabla_{X} \mathrm{PY}-\mathrm{P} \nabla_{X} Y, \quad \forall X, Y \in \Gamma(\mathrm{TM}), \\
\left(\tilde{\nabla}_{X} \mathrm{~F}\right) Y & =\nabla_{X} \mathrm{FY}-\mathrm{F} \nabla_{X} Y, \quad \forall X, Y \in \Gamma(\mathrm{TM}) .
\end{aligned}
$$

The structure vector field $\xi$ has been considered to be tangential to $M$ throughout this paper, else $M$ is simply anti-invariant [12. Since $\xi \in T M$, for any $X \in \Gamma(T M)$ by virtue of (2.5) and (2.6), we have

$$
\nabla_{X} \xi=X-\eta(X) \xi \text { and } \sigma(X, \xi)=0 .
$$

Making use of (2.4), (2.6), (2.7), (2.9), (2.10) and (2.12)-(2.14), we obtain

$$
\begin{aligned}
\left(\tilde{\nabla}_{X} P\right) Y & =p \sigma(X, Y)+A_{F Y} X+g(P X, Y) \xi-\eta(Y) P X \\
\left(\tilde{\nabla}_{X} F\right) Y & =f \sigma(X, Y)-\sigma(X, P Y)-\eta(Y) F X .
\end{aligned}
$$

A submanifold $M$ of an almost para contact metric manifold $\tilde{M}$ is said to be totally umbilical if

$$
\sigma(X, Y)=g(X, Y) H,
$$

where $H$ is the mean curvature vector of $M$. Further $M$ is totally geodesic if $\sigma(X, Y)=0$ and minimal if $\mathrm{H}=0$. 


\section{Slant submanifolds of an almost contact metric manifold}

For any $x \in M$ and $X \in T_{\chi} M$ such that $X, \xi$ are linearly independent, the angle $\theta(x) \in\left[0, \frac{\pi}{2}\right]$ between $\phi X$ and $T_{x} M$ is a constant $\theta$, that is $\theta$ does not depend on the choice of $X$ and $x \in M$. $\theta$ is called the slant angle of $M$ in $\tilde{M}$. Invariant and anti-invariant submanifolds are slant submanifolds with slant angle $\theta$ equal to 0 and $\frac{\pi}{2}$, respectively [5]. A slant submanifold which is neither invariant nor anti-invariant is called a proper slant submanifold.

We mention the following results for later use.

Theorem 3.1. [1] Let $M$ be a submanifold of an almost contact metric manifold $\tilde{M}$ such that $\xi \in \mathrm{TM}$. Then, $\mathrm{M}$ is slant if and only if there exists a constant $\lambda \in[0,1]$ such that

$$
P^{2}=-\lambda(I-\eta \otimes \xi)
$$

Further more, if $\theta$ is the slant angle of $\mathrm{M}$, then $\lambda=\cos ^{2} \theta$.

Corolary 1. [1] Let $\mathrm{M}$ be a slant submanifold of an almost contact metric manifold $\tilde{\mathrm{M}}$ with slant angle $\theta$. Then, for any $\mathrm{X}, \mathrm{Y} \in \mathrm{TM}$, we have

$$
\begin{gathered}
g(P X, P Y)=-\cos ^{2} \theta(g(X, Y)-\eta(X) \eta(Y)), \\
g(F X, F Y)=-\sin ^{2} \theta(g(X, Y)-\eta(X) \eta(Y)) .
\end{gathered}
$$

Theorem 3.2. Let $M$ be a totally umbilical slant submanifold of a para-Kenmotsu manifold $\tilde{M}$. Then either one of the following statements is true:

(i) $\mathrm{M}$ is invariant;

(ii) $\mathrm{M}$ is anti-invariant;

(iii) $\mathrm{M}$ is totally geodesic;

(iv) $\operatorname{dim} \mathrm{M}=1$;

(v) If $M$ is proper slant, then $\mathrm{H} \in \Gamma(\mu)$;

where $\mathrm{H}$ is the mean curvature vector of $\mathrm{M}$.

Proof. Suppose $M$ is totally umbilical slant submanifold, then we have

$$
\sigma(P X, P X)=g(P X, P X) H=\cos ^{2} \theta\left\{-\|X\|^{2}+\eta^{2}(X)\right\} H .
$$

By virtue of (2.6), one can get

$$
\cos ^{2} \theta\left\{-\|\mathrm{X}\|^{2}+\eta^{2}(\mathrm{X})\right\} \mathrm{H}=\tilde{\nabla}_{\mathrm{PX}} \mathrm{PX}-\nabla_{\mathrm{PX}} \mathrm{PX} .
$$

From (2.9), we have

$$
\cos ^{2} \theta\left\{-\|X\|^{2}+\eta^{2}(X)\right\} H=\tilde{\nabla}_{\mathrm{PX}} \phi X-\tilde{\nabla}_{\mathrm{PX}} \mathrm{FX}-\nabla_{\mathrm{PX}} \mathrm{PX} .
$$


Applying (2.7) and (2.12), we get

$$
\cos ^{2} \theta\left\{-\|\mathrm{X}\|^{2}+\eta^{2}(\mathrm{X})\right\} \mathrm{H}=\left(\tilde{\nabla}_{\mathrm{PX}} \phi\right) \mathrm{X}+\phi \tilde{\nabla}_{\mathrm{PX}} \mathrm{X}+\mathrm{A}_{\mathrm{FX}} \mathrm{PX}-\nabla_{\mathrm{PX}}^{\perp} \mathrm{FX}-\nabla_{\mathrm{PX}} \mathrm{PX} .
$$

Using (2.4) and (2.6), we obtain

$$
\begin{aligned}
\cos ^{2} \theta\left\{-\|\mathrm{X}\|^{2}+\eta^{2}(\mathrm{X})\right\} \mathrm{H}= & g(\phi \mathrm{PX}, \mathrm{X}) \xi-\eta(\mathrm{X}) \phi \mathrm{PX}+\phi\left(\nabla_{\mathrm{PX} X}+\sigma(\mathrm{X}, \mathrm{PX})\right) \\
& +\mathrm{A}_{\mathrm{FX}} \mathrm{PX}-\nabla_{\mathrm{PX}}^{\perp} \mathrm{FX}-\nabla_{\mathrm{PX}} \mathrm{PX} .
\end{aligned}
$$

From (2.9), (2.11), (2.18) and the fact that $X$ and PX are orthogonal vector fields on $M$, we arrive at

$$
\begin{aligned}
\cos ^{2} \theta\left\{-\|X\|^{2}+\eta^{2}(X)\right\} H= & -g(P X, P X) \xi-\eta(X) P^{2} X-\eta(X) F P X+P \nabla_{P X} X+F \nabla_{P X} X \\
& +A_{F X} P X-\nabla_{P X}^{\perp} F X-\nabla_{P X} P X .
\end{aligned}
$$

Then applying (3.1) and (3.2), we obtain

$$
\begin{aligned}
\cos ^{2} \theta\left\{-\|X\|^{2}+\eta^{2}(X)\right\} H= & \cos ^{2} \theta\left\{\|X\|^{2}-\eta^{2}(X)\right\} \xi+\cos ^{2} \theta \eta(X)\{X-\eta(X)\} \xi-\eta(X) F P X \\
& +P \nabla_{P X} X+F \nabla_{P X} X+A_{F X} P X-\nabla_{P X}^{\perp} F X-\nabla_{P X} P X
\end{aligned}
$$

Taking inner product with PX in (3.4), we get

$$
0=g\left(P \nabla_{P X} X, P X\right)+g\left(A_{F X} P X, P X\right)-g\left(\nabla_{P X} P X, P X\right) .
$$

By virtue of (3.2), the first term of (3.5) can be written as

$$
g\left(P \nabla_{P X} X, P X\right)=-\cos ^{2} \theta\left\{g\left(\nabla_{P X} X, X\right)-\eta(X) g\left(\nabla_{P X} X, \xi\right)\right\} .
$$

We simplify the third term of (3.5) as follows

$$
\begin{aligned}
g\left(\nabla_{P X} P X, P X\right) & =g\left(\tilde{\nabla}_{P X} P X, P X\right)=\frac{1}{2} P X g(P X, P X) . \\
& =\frac{1}{2} P X\left[-\cos ^{2} \theta\left\{\left(g(X, X)-\eta^{2}(X)\right)\right\}\right] \\
& =-\frac{1}{2} \cos ^{2} \theta[P X g(X, X)-P(X)(g(X, \xi) g(X, \xi))] \\
& =-\frac{1}{2} \cos ^{2} \theta[P X g(X, X)-2 \eta(X) P(X) g(X, \xi)] \\
& =-\frac{1}{2} \cos ^{2} \theta\left[2 g\left(\tilde{\nabla}_{P X} X, X\right)-2 \eta(X)\left\{g\left(\tilde{\nabla}_{P X} X, \xi\right)+g\left(X, \tilde{\nabla}_{P X} \xi\right)\right\}\right] .
\end{aligned}
$$

Using (2.5), (2.6), (3.6) and the fact that $X$ and PX are orthogonal vector fields on $M$, we derive

$$
\begin{aligned}
g\left(\nabla_{\mathrm{PX}} \mathrm{PX}, \mathrm{PX}\right)= & -\cos ^{2} \theta\left[\mathrm{g}\left(\nabla_{\mathrm{PX} X}, \mathrm{X}\right)-\eta(X) \mathrm{g}\left(\nabla_{\mathrm{PX}} X, \xi\right)\right. \\
& -\eta(X) \mathrm{g}(X, \mathrm{PX}-\eta(\mathrm{PX}) \xi)] \\
= & -\cos ^{2} \theta\left[\mathrm{g}\left(\nabla_{\mathrm{PX}} X, X\right)-\eta(X) \mathrm{g}\left(\nabla_{\mathrm{PX}} X, \xi\right)\right] \\
\rightarrow \mathrm{g}\left(\nabla_{\mathrm{PX}} \mathrm{PX}, \mathrm{PX}\right)= & g\left(\mathrm{P} \nabla_{\mathrm{PX} X}, \mathrm{PX}\right) .
\end{aligned}
$$


Using this fact in (3.5), we obtain

$$
0=g\left(A_{F X} P X, P X\right)=g(\sigma(P X, P X), F X) .
$$

As $M$ is totally umbilical slant, then from (2.18) and (3.2), we obtain

$$
0=-\cos ^{2} \theta\left\{\|X\|^{2}-\eta^{2}(X)\right\} g(H, F X) .
$$

Thus from (3.7), we conclude that either $\theta=\frac{\pi}{2}$ that is $M$ is anti-invariant which part (ii) or the vector field $X$ is parallel to the structure vector field $\xi$, i.e., $M$ is 1-dimensional submanifold which is fourth part of the theorem or $\mathrm{H} \perp \mathrm{FX}$, for all $\mathrm{X} \in \Gamma(\mathrm{TM})$, i.e., $\mathrm{H} \in \Gamma(\mu)$ which is the last part of the theorem or $H=0$, i.e., $M$ is totally geodesic which is (iii) or $F X=0$, i.e., $M$ is invariant which is part (i). This completes the proof of the theorem.

Theorem 3.3. Every totally umbilical proper slant submanifold of a para-Kenmotsu manifold is totally geodesic.

Proof. Let $M$ be a totally umbilical proper slant submanifold of a para-Kenmotsu manifold $\tilde{M}$, then for any $X, Y \in \Gamma(T M)$, we have

$$
\tilde{\nabla}_{X} \phi Y-\phi \tilde{\nabla}_{X} Y=g(\phi X, Y) \xi-\eta(Y) \phi X
$$

Using equations (2.6) and (2.9), we get

$$
\tilde{\nabla}_{X} P Y+\tilde{\nabla}_{X} F Y-\phi\left(\nabla_{X} Y+\sigma(X, Y)\right)=g(P X, Y) \xi-\eta(Y) P X-\eta(Y) F X
$$

Again from (2.6), (2.7) and (2.9), we obtain

$$
\begin{aligned}
g(P X, Y) \xi-\eta(Y) P X-\eta(Y) F X= & \nabla_{X} P Y+\sigma(X, P Y)-A_{F Y} \\
& +\nabla_{X}^{\perp} F Y-P \nabla_{X} Y-F \nabla_{X} Y-\phi \sigma(X, Y) .
\end{aligned}
$$

As $M$ is totally umbilical, then

$$
\begin{aligned}
g(P X, Y) \xi-\eta(Y) P X-\eta(Y) F X= & \nabla_{X} P Y+g(X, P Y) H-A_{F Y} X+\nabla_{X}^{\perp} F Y \\
& -P \nabla_{X} Y-F \nabla_{X} Y-g(X, Y) \phi H .
\end{aligned}
$$

Taking the inner product with $\phi \mathrm{H}$ in (3.8) and from the fact that $H \in \Gamma(\mu)$, we obtain

$$
g\left(\nabla \frac{\perp}{X} F Y, \phi H\right)=-g(X, Y)\|H\|^{2} .
$$

Applying (2.7) and the property of Riemannian connection the above equation takes the form

$$
g\left(F Y, \nabla \frac{1}{X} \phi H\right)=g(X, Y)\|H\|^{2} .
$$


Now for any $\mathrm{X} \in \Gamma(\mathrm{TM})$, we have

$$
\tilde{\nabla}_{X} \phi H=\left(\tilde{\nabla}_{X} \phi\right) H+\phi \tilde{\nabla}_{X} H .
$$

Using the fact $H \in \Gamma(\mu)$ and by virtue of (2.4), (2.7) and (2.9), we obtain

$$
-A_{\phi H} X+\nabla \frac{\perp}{X} \phi H=-P A_{H} X-F A_{H} X+\phi \nabla_{X}^{\perp} H .
$$

Also for any $X \in \Gamma(T M)$, we have

$$
g\left(\nabla_{X}^{\perp} H, F X\right)=g\left(\tilde{\nabla}_{X} H, F X\right)=-g\left(H, \tilde{\nabla}_{X} F X\right) .
$$

Using (2.9), we obtain

$$
g\left(\nabla_{X}^{\perp} H, F X\right)=-g\left(H, \tilde{\nabla}_{X} \phi X\right)+g\left(H, \tilde{\nabla}_{X} P X\right) .
$$

Further from (2.6) and (2.12), we derive

$$
g\left(\nabla_{X}^{\frac{1}{X}} H, F X\right)=-g\left(H,\left(\tilde{\nabla}_{X} \phi\right) X\right)-g\left(H, \phi \tilde{\nabla}_{X} X\right)+g(H, \sigma(X, P X)) .
$$

Using (2.4) and (2.18), the first and last term of right hand side of the above equation are identically zero and hence by (2.3), the second term gives

$$
g\left(\nabla_{X}^{\perp} H, F X\right)=g\left(\phi H, \tilde{\nabla}_{X} X\right) .
$$

Again by using (2.6) and (2.18), we obtain

$$
g\left(\nabla \frac{1}{X} H, F X\right)=g(\phi H, H)\|X\|^{2}=0 .
$$

This means that

$$
\nabla_{\mathrm{X}}^{\perp} \mathrm{H} \in \Gamma(\mu) .
$$

Now, taking the inner product in (3.10) with $F Y$ for any $Y \in \Gamma(T M)$, we get

$$
g\left(\nabla \frac{1}{X} \phi H, F Y\right)=-g\left(F A_{H} X, F Y\right)+g\left(\phi \nabla \frac{1}{X} H, F Y\right) .
$$

Using (3.11) and from (3.3) and (3.9), we obtain

$$
g(X, Y)\|H\|^{2}=\sin ^{2} \theta\left\{g\left(A_{H} X, Y\right)-\eta(Y) g\left(A_{H} X, \xi\right)\right\} .
$$

Hence by (2.8) and (2.18), the above equation reduces to

$$
g(X, Y)\|H\|^{2}=\sin ^{2} \theta\left\{g(X, Y)\|H\|^{2}-\eta(Y) g(\sigma(X, Y), H)\right\} .
$$

Since for a para-Kenmotsu manifold $\tilde{M}, \sigma(X, \xi)=0$ for any $X$ tangent to $\tilde{M}$, thus we obtain

$$
g(X, Y)\|H\|^{2}=\sin ^{2} \theta\left\{g(X, Y)\|H\|^{2} .\right.
$$

Therefore, the above equation can be written as

$$
\cos ^{2} \theta g(X, Y)\|H\|^{2}=0
$$

Since $M$ is proper slant submanifold, thus from (3.14) we conclude that $H=0$, i.e., $M$ is totally geodesic in $\tilde{M}$. This completes the proof. 


\section{References}

[1] A.M. Blaga, Invariant, anti-invariant and slant submanifolds of a para-Kenmotsu manifold, BSG Proceedings, 24 (2017), 19-28.

[2] A.M. Blaga, Eta-Ricci solitons on para-Kenmotsu manifolds, Balkan Journal of Geometry and Its Applications, 20(1) (2015), 1-13.

[3] J.L. Cabrerizo, A. Carriazo and L.M. Fernandez, Slant submanifolds in Sasakian manifolds, Glasgow Math. J., 42 (2000), 125-138.

[4] B.Y. Chen, Slant immersions, Bull. Aust. Math. Soc., 41 (1990), 135-147.

[5] B.Y. Chen, Geometry of slant submanifolds, Katholieke Universiteit Leuven, (1990).

[6] P. Dacko and Z. Olszak, On weakly para-cosymplectic manifolds of dimension 3, J. Geom. Phys., 57 (2007), 561-570.

[7] R.S. Gupta, S.M. Khursheed Haider and M.H. Shahid, Slant submanifolds of a Kenmotsu manifold, Radovi Matematicki, Vol. 12 (2004), 205-214.

[8] R.S. Gupta and P.K. Pandey, Structure on a slant submanifold of a Kenmotsu manifold, Differential Geometry - Dynamical Systems, 10 (2008), 139-147.

[9] S. Ivanov, D. Vassilev and S. Zamkovoy, Conformal paracontact curvature and the local flatness theorem, Geom. Dedicata, 144 (2010), 79-100.

[10] K. Kenmotsu, A class of almost contact Riemannian manifolds, Tohoku Math. J., 24 (1972), 93-103.

[11] M.A. Khan, S. Uddin and K. Singh, A classification on totally umbilical proper slant and hemislant submanifolds of a nearly trans-Sasakian manifold, Differential Geometry - Dynamical Systems, 13 (2011), 117-127.

[12] A. Lotta, Slant submanifolds in contact geometry, Bull. Math. Soc. Roum., 39 (1996), 183-198.

[13] A. Lotta, Three dimensional slant submanifolds of K-contact manifolds, Balkan J. Geom. Appl., 3(1) (1998), 37-51.

[14] M.S. Siddesha and C.S. Bagewadi, On slant submanifolds of $(k, \mu)$-contact manifold, Differential Geometry-Dynamical Systems, 18 (2016), 123-131.

[15] M.S. Siddesha and C.S. Bagewadi, Semi-slant submanifolds of $(k, \mu)$-contact manifold, Commun. Fac. Sci. Univ. Ser. A 1 Math. Stat., 67(2) (2017), 116-125. 
[16] B.B. Sinha and K.L. Sai Prasad, A class of almost para contact metric manifolds, Bull. Cal. Math. Soc., 87 (1995), 307-312.

[17] J. Welyczko, Slant curves in 3-dimensional normal almost paracontact metric manifolds, Mediterr. J. Math., DOI 10.1007/s00009-013-0361-2, 2013.

[18] S. Zamkovoy, Canonical connections on paracontact manifolds, Ann. Global Anal. Geom., 36(1) (2008), 37-60. 